\title{
EL SENTIDO Y EL SIGNIFICADO ATRIBUIDO A LAS EMOCIONES POR EL PROFESORADO EN FORMACIÓN DE LA UNIVERSIDAD PEDAGÓGICA Y TECNOLÓGICA DE COLOMBIA
}

\author{
Rafael Enrique Buitrago Bonilla \\ Universidad Pedagógica y Tecnológica de Colombia, Tunja \\ Aida Karina Avila Moreno \\ Universidad Pedagógica y Tecnológica de Colombia, Sogamoso \\ Ruth Nayibe Cárdenas Soler \\ Universidad Pedagógica y Tecnológica de Colombia, Tunja
}

\begin{abstract}
RESUMEN: Una educación de calidad no solo implica la formulación coherente de planes de estudio, sino que debe contemplar otros aspectos en la formación del profesorado, como las competencias emocionales, siendo un proceso que redundará en el mismo sistema educativo. Es así como este artículo presenta el sentido y el significado que los maestros en formación de la Facultad de Ciencias de la Educación en la Universidad Pedagógica y Tecnológica de Colombia le asignan a las emociones, como resultado de una investigación de tipo cualitativo que utilizó la metodología de estudio de caso y la técnica de entrevista a profundidad. Los participantes fueron nueve mujeres y ocho hombres de octavo o noveno semestre de 10 carreras universitarias de formación de maestros en distintas áreas. La investigación realizada puso de manifiesto que estos programas profesionales no integran de manera estructurada el estudio de las emociones y que el manejo emocional inadecuado puede tener un impacto negativo en el proceso educativo. De igual manera, resultó importante considerar la interdependencia entre lo intrapersonal e interpersonal y la relación entre los sentidos atribuidos a las emociones por parte de los participantes y los principales modelos de inteligencia emocional.
\end{abstract}

PALABRAS CLAVE: Significado de las emociones, sentido atribuido a las emociones, Maestros en Formación.

\section{THE SENSE AND MEANING ATTRIBUTED}

\section{TO EMOTIONS BY TEACHERS TRAINING AT PEDAGOGICAL AND} TECHNOLOGICAL UNIVERSITY OF COLOMBIA

ABSTRACT: Quality education not only involves the formulation of curriculum but must consider other aspects into teacher training, such as emotional 
competencies, because it will improve the education system. This paper is a result of a study of qualitative typology that highlights the sense and meaning that training teachers give to emotions, on the Faculty of Education Sciences at the Pedagogical and Technological University of Colombia. The data collection technique used were case study and in-depth interview. Nine men and ten women students from different areas (8th and 9th semester) of 10 training teacher programs participated in this research. In the research was evident that training teacher programs do not include the study of emotions and that inadequate emotional management can have a negative impact on the educational process. Likewise, it was important to consider the interdependent relationship between intrapersonal and interpersonal topics and the concordance between meaning attributed to the emotions from participants and the main models of emotional intelligence.

KEYWORDS: Meaning of emotions, sense attributed to emotions, training teachers.

Recibido: 22/03/2016

Aceptado: 14/07/2016

Correspondencia: Rafael Enrique Buitrago Bonilla, Universidad Pedagógica y Tecnológica de Colombia, Avenida Central del Norte 39-115, Sede Central Tunja. Boyacá (Colombia). Email: lafaremus@gmail.com.

\section{INTRODUCCIÓN}

Las emociones están relacionadas con las respuestas hacia sí mismo, hacia los demás y hacia el contexto, es por ello que afectan la vida personal, escolar y profesional, es decir, inciden de manera relevante en la satisfacción y bienestar profesional, así como en la eficacia social (Carpena y Darder, 2010). Por lo tanto, además de la incidencia reciproca que tienen las emociones en maestros y alumnos es preciso resaltar que generan un clima emocional en la escuela, de la cual hacen parte los directivos, padres de familia y demás personas y profesionales que integran el contexto escolar (Hargreaves, 2000).

En la práctica educativa tanto el afecto como las emociones son imprescindibles, ya que ellas inciden en el desarrollo del estudiantado y del profesorado, así como en los ambientes y metodologías implementadas en el aula. En consecuencia, es fundamental que durante la formación del profesorado se aborde la teoría de las emociones, junto con el desarrollo de habilidades emocionales, la gestión emocional y la dimensión emocional de la práctica educativa (Abarca, Marzo y Sala, 2002).

Desde la perspectiva de Palomera, Fernández-Berrocal y Bracket (2008), es indispensable que los programas de formación docente integren de manera explícita en sus planes de estudio el aprendizaje de competencias emocionales y no queden limitadas a ser competencias transversales. Al lograrlo, el profesorado será más expresivo, respetuoso, auto motivado y empático, aspectos que optimizan su labor educativa (Calle, Remolina y Velásquez, 2011). 
Las emociones tienen gran importancia en la formación docente, tanto en su abordaje teórico como práctico, es así como su incidencia en la enseñanza y en el aprendizaje deben estar presentes durante todo el proceso educativo (Abarca et al., 2002; Macazaga, Vaquero y Gómez, 2013; Postareff y Lindblom-Ylänne, 2011). Es decir que tanto la formación inicial del profesorado, como la formación continuada, son fundamentales para que estos profesionales tengan un alto rendimiento, un óptimo desempeño y un adecuado bienestar personal (Palomera et al., 2008). Sin embargo, lograr una adecuada educación emocional requiere que el profesorado comprenda su importancia (Bisquerra y Pérez, 2012).

En consecuencia, los requerimientos de los actuales contextos escolares implican un nuevo perfil para los maestros, que les permita ofrecer respuesta a las actuales condiciones y a los permanentes cambios sociales (Buitrago y Herrera, 2013). Para enfrentar este reto es imprescindible que el profesorado trascienda las acciones individuales e implemente en su práctica cotidiana el trabajo en red. Todo esto, teniendo en cuenta que los actuales contextos escolares en los que se desempeña el profesorado suscitan una gran cantidad de emociones y sentimientos, que cuando no logran gestionarse, suelen generar malestar o desajuste emocional (Carpena y Darder, 2010). Es decir que las competencias emocionales se establecen como competencias básicas para la vida, que permiten a los maestros abordar con mayor solvencia las complejidades que se presentan en el ámbito educativo (Bisquerra, 2000, 2005).

Evidentemente, el profesorado integra diferentes estrategias para los procesos de enseñanza aprendizaje, algunas de ellas relacionadas con aspectos emocionales y en algunos casos de manera no consciente. En este sentido en 2016, Habók y Nagy encontraron en su estudio que el profesorado utilizaba de manera preferente metodologías fundamentadas en proyectos, especialmente en el aprendizaje basado en problemas, dando realce a la motivación, sin embargo, sus hallazgos mostraron que los maestros no aprovechaban el potencial de las emociones y el uso de TIC, dejando la educación social y emocional así como la evaluación en un segundo lugar. Desde otra perspectiva Zee, de Jong y Koomen (2016) evidenciaron que los profesores suelen tener una mayor sensación de autoeficacia con los estudiantes que presentan mejores habilidades prosociales, no obstante, estos docentes suelen dedicar menos tiempo a estos alumnos y por lo general no les reconocen y estimulan estas conductas positivas.

Bajo esa tesitura, la formación del profesorado debe integrar aspectos como el bienestar individual subjetivo, que desde la perspectiva de Hernández (2002) se define como "una evaluación global, hecha por uno mismo y sobre uno mismo, dentro de un periodo amplio de tiempo, acerca de la satisfacción con la vida" (p. 30). Sumado a ello, la automotivación es relevante para el profesorado, ya que le permite mantener la ilusión e interés hacia su trabajo, a pesar de las circunstancias que se presentan en su entorno (Bazarra, Casanova y García, 2004). Al respecto, Hué (2012) enfatiza en que las competencias emocionales del maestro son las que éste imprime en sus estudiantes, en un clima de motivación o desmotivación, influenciando además su trabajo didáctico y bienestar docente.

Propiciar experiencias de aprendizaje emocional posibilitan el disfrute de los procesos cognitivos, en especial desde el desarrollo e implementación del pensamiento 
crítico y la auto-regulación como proceso metacognitivo (Ranellucci, Hall y Goetz, 2015). Aun cuando no se ha determinado de manera suficiente la incidencia de las emociones en los procesos de enseñanza-aprendizaje, es claro que el vínculo afectivo que se genera entre el alumno y el conocimiento, el cual fortalece y mejora el aprendizaje (Andrade, 2016). A su vez, las emociones de los profesores tienen relación con su calidad en la enseñanza, el optimismo académico, la esperanza, la responsabilidad personal, la motivación, el compromiso y la satisfacción laboral (Eren, 2014).

Otro aspecto imprescindible en el ejercicio de la docencia es la creatividad, la cual debería también estar presente durante el proceso de formación del profesorado. La creatividad tiene una relación directa con las emociones debido a que el proceso creativo integra un sin número de emociones tales como entusiasmo, alegría, deseo, ira, tristeza, frustración, euforia, etc. Por lo tanto, es importante fortalecer la regulación emocional para lograr el paso entre el potencial creativo y el logro creativo (Ivcevic y Brackett, 2015). De esta forma, la regulación emocional permite hacer frente a la pérdida del interés en una actividad específica, superando la mortificación creativa (Beghetto, 2014).

Ivcevic y Brackett (2015) manifiestan que la gestión emocional es relevante para abordar aspectos intrapersonales como los sentimientos generados por la evaluación negativa de un trabajo propio o la euforia que puede llegar a ser abrumadora y que es suscitada, en muchas ocasiones, por el éxito al lograr un producto creativo; pero también en aspectos interpersonales como la comunicación afectiva con colegas o estudiantes. De ahí que sea indispensable que los programas de formación de maestros aborden las demandas emocionales de los procesos de enseñanza-aprendizaje, al igual que el desarrollo de habilidades de regulación emocional (Relojo, Pilao y de la Rosa, 2015).

Lo cierto es que el profesorado hace frente a diferentes emociones en su ejercicio profesional cotidiano, por ejemplo, el gusto por lo que se hace y cómo se hace en el aula es un aspecto determinante para su satisfacción y autoimagen percibida (Andrade, 2016). De igual modo el profesorado experimenta de manera periódica sentimientos de frustración, desánimo y desilusión con la enseñanza (Eren, 2014).

Se puede afirmar que el abordaje de la teoría y el desarrollo de habilidades emocionales son fundamentales en la formación de maestros, para fortalecer el ámbito intrapersonal y los vínculos relacionales positivos que el profesorado debe utilizar en el ejercicio de su profesión. En este sentido, es imprescindible el estudio de los "hot moments" que se refieren a aquellos instantes repentinos en los que las emociones generan altos niveles de tensión, presión o conflicto, en muchas ocasiones desencadenados por apreciaciones respecto a temáticas que pueden llegar a ser álgidas y que termina afectando los procesos de enseñanza-aprendizaje (Harlap, 2014; Hughes, Huston y Stein, 2010; Warren, 2006). En esta dirección Sue, Lin, Torino, Capodilupo y Rivera (2009) enfatizan en la relevancia de las microagresiones, que son acciones intencionales o no intencionales que se presentan en la cotidianidad de las aulas, derivadas por lo general de la diversidad de las personas que asisten a ellas, y que por lo general afectan la dignidad individual o grupal (raza, género, etc.).

Hay una relación entre las ideas y las creencias de los profesores sobre las emociones, sus prácticas y enseñanzas socioemocionales, por lo tanto, en gran medida, 
en su incidencia en el aprendizaje y el comportamiento (Garner, Moses y Waajid, 2009; Zinsser, Shewark, Denham y Curby, 2014). De esta forma, se considera relevante que en los programas de formación de maestros trabajen las habilidades y gestión emocional, además del abordaje de la teoría de las emociones y la inteligencia emocional, la empatía, las técnicas de regulación emocional y el comportamiento social y pro social.

La formación profesional del profesorado se orienta a la mejora de los procesos de enseñanza-aprendizaje, desde la consolidación de seres humanos críticos, creativos y autónomos, con una profunda responsabilidad intrapersonal e interpersonal que disminuya los hábitos mecánicos y propenda tanto por la flexibilidad como por la adaptabilidad (Macazaga et al., 2013), donde también es fundamental la temática referente a la gestión de las emociones.

El déficit de habilidades emocionales incide tanto en el profesorado como en el estudiantado. Es así como un alto porcentaje de las bajas laborales se derivan de factores y estados emocionales. En los últimos años se ha incrementado el burnout (también Ilamado síndrome de desgaste profesional u ocupacional), el cual implica agotamiento y frustración docente, desmotivación, depresión y absentismo, generando en muchos casos alteraciones psicofisiológicas como cefaleas, insomnio, ulceras y alergias, entre otras (Durán, Extremera, Rey, Fernández-Berrocal y Montalbán, 2006; Extremera, Fernández-Berrocal y Durán, 2003; Mesa et al., 2002; Palomera, Gil-Olarte y Brackett, 2006). En muchas ocasiones, estos estados son producto de carencias empáticas entre colegas, así como de ambientes negativos (Bakker, Westman y Schaufeli, 2007). En cuanto a los alumnos, se observa baja tolerancia a la frustración, conductas inapropiadas derivadas de la ira, acoso, violencia, maltrato, abuso de alcohol y sustancias psicoactivas, entre otras (Bisquerra y Pérez, 2012).

El estrés laboral es un fenómeno complejo en el mundo actual, que se establece como un factor relevante en el contexto de la salud y que incide de manera creciente en el profesorado, sin embargo, los programas de formación docente no suelen abordar la gestión del mismo, es por ello que la capacitación en el tema emocional y las técnicas de auto-eficacia son alternativas pertinentes. En consecuencia, las habilidades emocionales que los docentes deben desarrollar en sus procesos formativos, en el ámbito intrapersonal e interpersonal, posibilitan la reducción del estrés en la enseñanza y mejoran el bienestar psicológico, debido a que permite al profesorado relacionarse de mejor manera con las demás personas (Jiboku, 2015). La calidad de estos vínculos está asociada al desarrollo de habilidades para percibir las diversas perspectivas y el desasosiego de los demás, logrando así relaciones sociales significativas y saludables (Castro y Zautra, 2016).

La insatisfacción laboral, el agotamiento emocional y diversos problemas de salud se derivan, en muchas ocasiones, de situaciones de estrés (Hochschild, 1990; Maslach, 1982; Morris y Feldman, 1996; Schaubroeck y Jones, 2000). Por esta razón, cuando el profesorado se enfrenta a episodios prolongados de estrés se genera una disminución en sus habilidades de razonamiento, afrontamiento y decisión (Hué, 2012). Ese estrés suele aparece en los momentos en que el profesorado se siente presionado por el exceso de trabajo (Servan-Scheiber, 2004). No obstante, es necesario advertir que el trabajo también se constituye en una experiencia vital 
y significativa para muchas personas, que da sentido y sirve de motor para el desarrollo de las demás áreas de la vida (Avila y Castañeda, 2015).

Brotheridge y Grandey (2002) plantean que la docencia es una de las profesiones más estresantes, debido a la interacción social cotidiana con colegas, estudiantes, padres de familia, directivos y demás miembros de la comunidad educativa, en donde la empatía y la regulación emocional son protagonistas. En esta misma línea de pensamiento, Emmer (1994) señala que por lo general el profesorado está expuesto con mayor frecuencia a emociones y entornos negativos, mientras que Marchesi y Díaz (2007) exponen, a partir de un estudio, que a pesar de que la mayor parte del profesorado se sentía complacido con su profesión la mayoría $(74,5 \%)$ tenía la percepción de que la profesión docente había empeorado y por ende se había aumentado el malestar del colectivo. Sumado a ello, Klusmann, Richter y Lüdtke (2016) exponen que el desgaste profesional, el agotamiento emocional y el estrés de los maestros están relacionados con el bajo rendimiento académico del estudiantado.

Desde otra perspectiva, cuando los maestros se enfrentan a un alto número de estudiantes difíciles pueden generar actitudes negativas hacia ellos, debido en gran medida a que pueden llegar a sentirse incapaces para enseñar o apoyar afectivamente a sus estudiantes, alejándose, por lo general, de ellos (Zee et al., 2016). La práctica docente profesional está asociada a procesos permanentes de evaluación y autoevaluación, los cuales asumen, en la mayoría de los casos, la excelencia como pauta de contrastación, parámetro que puede llegar a incidir en el bajo concepto que de sí mismo tiene un alto porcentaje de docentes, sobre todo en el ámbito de la educación secundaria y superior. Estas circunstancias generan que la mayoría del profesorado, no confiera una valoración adecuada a sus propias habilidades y capacidades (Hué, 2012). Por lo anteriormente mencionado se hace necesario el abordaje de las emociones y la inteligencia emocional en los programas de formación docente, debido a que éstas inciden de manera directa en la salud mental y física de los sujetos (Calle et al., 2011; Davidson, 2003; Ekman, 2003).

Desde la perspectiva de Brady et al. (2016) se pueden asumir distintas estrategias para hacer frente al estrés y a la adversidad, entre ellas la motivación desde la autoafirmación, la cual puede llegar a tener un impacto positivo a largo plazo, debido a que los efectos que se logran se repiten en las experiencias subjetivas y la humanización de las relaciones. Todo esto implica el reconocimiento de la individualidad de los demás en cuanto a sus emociones, pensamientos y perspectivas, consiguiendo vínculos positivos más fuertes y duraderos, junto con mayor bienestar individual y colectivo (Castro y Zautra, 2016).

Para finalizar, se puede señalar que aún son pocos los estudios que abordan aspectos como la salud y el bienestar de los docentes (Cornejo y Quiñonez, 2007), hecho que sugiere la necesidad de incrementar las investigaciones enfocadas en los aspectos afectivos de la formación de maestros (Hodgen y Askew, 2007), especialmente en educación superior (Postareff y Lindblom-Ylänne, 2011), atendiendo a sus propias percepciones y permitiéndoles que expresen su sentir. Por lo tanto, la presente investigación buscó cuestionar los sentidos y significados que los maestros en formación de la Facultad de Ciencias de la Educación de la Universidad Pedagógica y Tecnológica de Colombia - UPTC le concedían a las emociones. 


\section{Método}

Para el desarrollo de la presente investigación se utilizó como herramienta metodológica el estudio de caso, definido por Rodríguez, Gil y García (1996) como el proceso de indagación caracterizado por un análisis minucioso, comprensivo, profundo y procedimental del objeto de interés. Para el presente estudio se utilizó la tipología tres, un caso con múltiples unidades de análisis (Yin, 1994, 1999).

\section{Participantes}

En el estudio participaron 17 estudiantes de Licenciatura (9 mujeres y 8 hombres), es decir, de programas profesionales de formación docente en distintas áreas de la Facultad de Ciencias de la Educación en la UPTC. Los estudiantes pertenecían a octavo y noveno semestre. El tipo de muestra utilizado fue no probabilístico por conveniencia (Hernández, Fernández-Collado y Baptista, 2006) y se tuvo en cuenta, para decidir el número final de entrevistas, la saturación de categorías, es decir, el momento en que no se encontraron categorías nuevas, significativas o diferentes (Coleman y Unrau, 2005).

\section{Técnica de Recolección de Información}

La técnica de recolección de información utilizada fue la entrevista a profundidad, tomando como base un protocolo previo, estructurado a partir de dos categorías orientadoras y distintos ejes de indagación, con base en los cuales se elaboraron 44 preguntas. Una vez definido el protocolo de entrevista, el mismo fue enviado a cuatro profesores investigadores para su revisión y de acuerdo con sus sugerencias se realizaron los ajustes respectivos. Las dos categorías orientadoras fueron definidas como:

a. Sentido atribuido a las emociones. La interpretación personal que los participantes atribuyen a las emociones a partir de elementos psicológicos, históricos, culturales educativos y sociales.

b. Formación inicial del profesorado. Proceso educativo universitario de pregrado para la formación de los profesionales que ejercerán la actividad docente, para fortalecer el rendimiento y calidad del aprendizaje en el alumnado.

Durante el proceso de diseño y construcción del protocolo de entrevista, la evaluación del mismo y la posterior implementación con los estudiantes, se tuvo en cuenta la reactividad, al igual que las tendencias y sesgos de los participantes y de los investigadores. La dependencia, es decir fiabilidad cualitativa y la credibilidad, validez interna cualitativa (Hernández et al., 2006), se llevó a cabo a través de la triangulación de investigadores, la cual permitió determinar la coherencia de los análisis y resultados de varios investigadores a partir de los mismos datos (Franklin y Ballan, 2005). La triangulación de datos (utilización de diversas fuentes de estudio), se basó en la información que provenía de ocho programas de licenciatura diferentes, es decir, de formación de maestros en: Artes Plásticas, Ciencias Naturales y Educación Ambiental, Ciencias Sociales, Educación Física, Idiomas Modernos, Lenguas Extranjeras, Matemáticas, Música, Preescolar y Psicopedagogía. 


\section{Procedimiento}

De recogida de información. Para desarrollar la investigación, además del consentimiento informado de cada participante, se solicitó la autorización de los directores de cada uno de los programas de formación del profesorado de la UPTC. Las entrevistas a los estudiantes fueron realizadas por 7 investigadores durante 2015, bajo principios de cautela y coherencia.

De análisis de datos. Para el análisis de la información se hizo uso del programa informático ATLAS TI para Windows (versión 7). Dicho proceso se llevó a cabo con tres niveles de análisis, de acuerdo con la propuesta metodológica de la teoría fundamentada de Corbin y Strauss (2002): codificación abierta (proceso analítico por medio del cual se identifican los conceptos y se descubren en los datos sus propiedades y dimensiones), codificación axial (proceso para relacionar las categorías con sus subcategorías) y codificación selectiva (proceso para integrar las categorías principales y estructurar teoría).

\section{Resultados}

La Facultad de Ciencias de la Educación de la Universidad Pedagógica y Tecnológica de Colombia es la encargada de formar al profesorado que dará respuesta al sistema educativo colombiano en cada uno de sus niveles, estos son: preescolar, educación básica primaria, educación básica secundaria y educación media. Para ello, la Facultad ofrece 12 licenciaturas, que son programas profesionales de pregrado: Artes Plásticas, Ciencias Naturales y Educación Ambiental, Ciencias Sociales, Educación Física, Filosofía, Idiomas Modernos, Informática Educativa, Lenguas Extranjeras, Matemáticas, Música, Preescolar y Psicopedagogía.

El primer aspecto destacable que arrojó el estudio se refiere a que el profesorado en formación reconoce que las emociones juegan un papel relevante en su desarrollo personal y de manera particular en la elección de la docencia como profesión (ver figura 1).

En este sentido, tal y como se explicita en la figura 2, los participantes dieron diversos significados a las emociones, siendo su función de desarrollo intrapersonal e interpersonal la que tuvo mayor énfasis. Además de ello, se resalta que las emociones son parte constitutiva del ser humano, que tienen relación con los estados anímicos y las acciones, que operan de manera tanto positiva como negativa y que inciden en los procesos de enseñanza-aprendizaje.

Al respecto, entre algunas apreciaciones de los participantes en relación con las emociones se destaca: son "realmente importantes, tanto en mi desarrollo como en el de la sociedad" (M.F. 1); o "yo creo que las emociones nos hacen como personas, nos hacen ser las personas que somos" (M.F. 16)

Desde la perspectiva de la incidencia de las emociones en la educación, fueron cinco los aspectos señalados, los cuales se orientan a la estabilidad asumida como el bienestar de estudiantes y profesores; al ambiente que se puede llegar a generar en el aula y en la institución; al hecho de facilitar el aprendizaje y además lograr que sea significativo; $y$, finalmente, a la incidencia e impacto positivo y negativo que las emociones pueden llegar a suscitar. A propósito de este último aspecto, resulta relevante decir aquí que un buen número de estudiantes hicieron referencia a este criterio (ver Figura 3). 


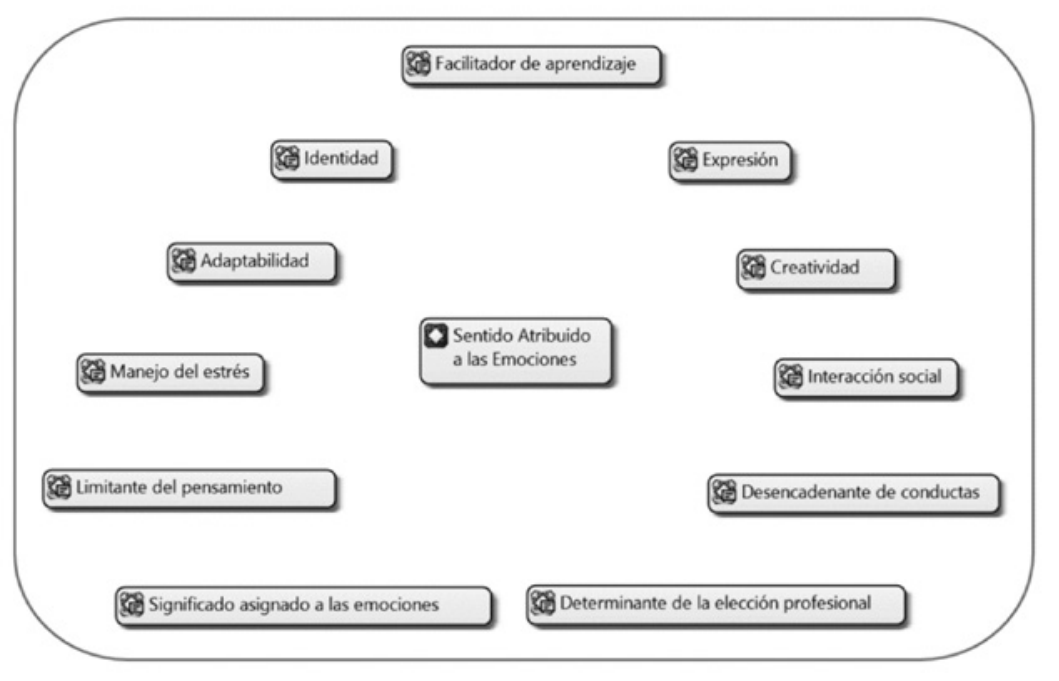

Figura 1. Sentido que los Maestros en Formación atribuyen a las emociones

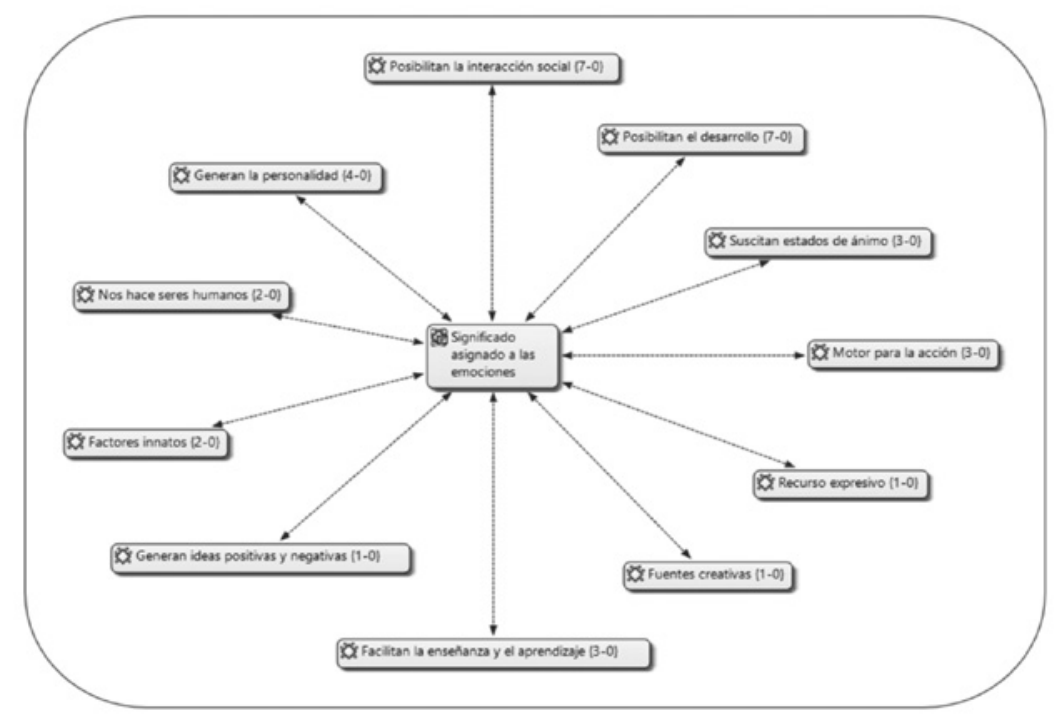

Figura 2. Significado que los Maestros en Formación confieren a las emociones

Al referirse a los procesos de apropiación del conocimiento, los estudiantes señalaron "sí, inciden las emociones en el proceso enseñanza aprendizaje, porque cuando a uno le enseñan algo que no le gusta va a generar una emoción fea" (M.F. 4); o "Inciden bastante porque hacen parte de lo que uno quiere y lo que a uno le gusta, la emoción permite que si tú estás feliz y tú quieres aprender vas a generar gusto por ese aprendizaje" (M.F. 17). En cuanto a los impactos negativos que las emociones tienen, plantearon "Los profesores o los compañeros no tienen en cuenta los sentimientos y uno se siente aislado" (M.F. 9). 


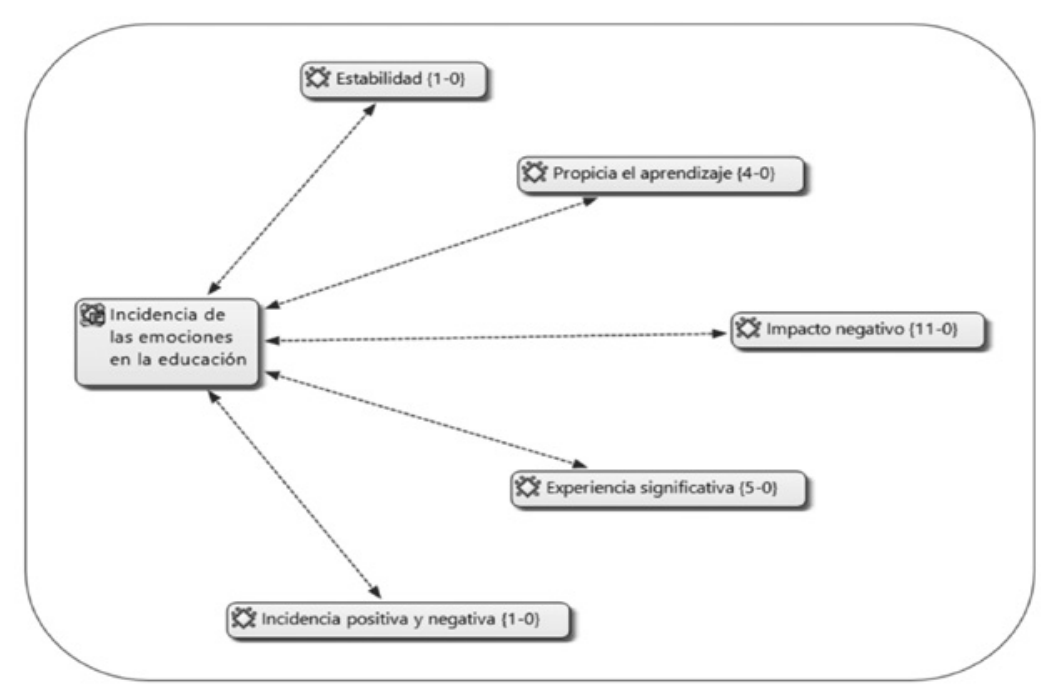

Figura 3. Incidencia de las emociones en la educación

En la Figura 4 se explicitan los seis criterios señalados por los estudiantes respecto al abordaje de teorías o temáticas relacionadas con las emociones. De estos criterios se puede señalar que hay tres tipos de perspectivas, la primera se refiere al trabajo superfluo, confuso o inexistente, siendo este aspecto el de mayor recurrencia por parte de los participantes. La segunda perspectiva corresponde al trabajo ocasional y la tercera al abordaje sistemático, estructurado y permanente. En este sentido los maestros en formación señalaron: "No, realmente es muy poco, poco, realmente nada" (M.F. 1).

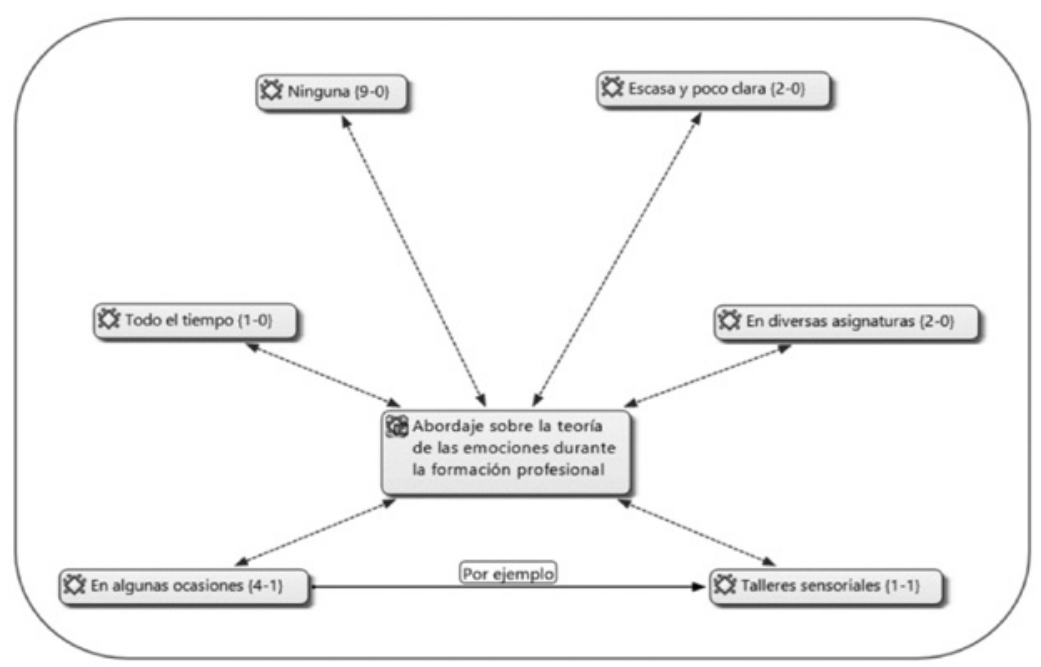

Figura 4. Perspectiva de los Maestros en Formación (M.F.) sobre el abordaje de la teoría de las emociones durante su formación profesional 


\section{Discusión}

El sentido atribuido a las emociones que exponen los estudiantes participantes del presenten estudio pone de manifiesto la importancia que le confieren a las emociones en su vida, incluida lógicamente la elección de su profesión, en este caso la educación. Las categorías emergentes en el estudio presentan concordancia con las planteadas en el modelo de habilidad de Mayer y Salovey (Brackett y Salovey, 2006; Mayer y Salovey, 1997), el modelo de inteligencia social y emocional de Bar-On $(1997,2006)$ y el modelo de competencias emocionales centradas en el lugar de trabajo de Goleman (Boyatzis, Goleman y Rhee, 2000; Goleman, 1998, 2001), de esta forma, es destacable señalar que los maestros en formación que participaron en el estudio, en relación con su propio desarrollo emocional y su conocimiento teórico, se perciben ansiosos y con miedo al estar cerca de concluir su proceso formativo y próximos a iniciar su ejercicio profesional.

Respecto al significado que los maestros en formación asignan a las emociones se puede afirmar que están en correspondencia con los planteamientos de Carpena y Darder (2010), quienes destacan la importancia de la interdependencia de habilidades intrapersonales (autoconocimiento, autoestima y gestión personal interna) e interpersonales (vínculos, encuentros con el otro y empatía) para alcanzar el desarrollo emocional y la ética solidaria; permitiendo además, tal y como lo señala la neurociencia, una interacción de los ámbitos corporales, emocionales, conductuales y cognitivos, posibilitando, a su vez, una relación armónica con las demás personas y consigo mismo.

Algunas de las apreciaciones de los maestros en formación respecto a las emociones fueron: "Las emociones para mi significan los estados de ánimo en los que uno está, los que uno demuestra" (M.F. 6). Por lo tanto "Las emociones deberían ser algo que desde casa se trabaje, y cuidar las emociones también, porque a veces uno se Ilena de emociones negativas, entonces ahí surgen problemas muy serios..." (M.F. 3).

Para los participantes del estudio las emociones juegan un papel relevante en la interacción con los demás. "Yo creo que a una persona sin emociones, le resulta bastante difícil desenvolverse en una dinámica educativa o social" (M.F. 12). En este sentido, la empatía también es contemplada por el profesorado en formación y presenta relación con los criterios propuestos por Herrera, Buitrago y Avila (2016), 'Los sentimientos juegan un papel muy importante, tanto en demostrar las cosas como en observar y percibir lo que las demás personas quieren saber o quieren aprender" (M.F. 1).

En los relatos de los participantes se hacen planteamientos que se relacionan con el concepto de adaptabilidad propuesto por Bar-On (1997, 2006):

...uno como docente tiene que ser muy paciente porque uno está enfrentando diferentes poblaciones, entonces las emociones juegan un papel muy importante, porque a veces hay que tratar a la persona que llega muy feliz a clase, muy brava, con depresión, uno como docente tiene que estar preparado para todos los casos, entonces son muy importantes ya que uno como docente tiene que saber llegar a todos los estudiantes y más con las generaciones que van cambiando y se vuelven más complicada... (M.F. 16).

Además, es importante resaltar la relación que se explicita entre emociones y creatividad. "Cuando uno está bien, tiene muchas ganas de hacer todo, le quedan cosas geniales, a veces uno quisiera hacer más..." (M.F. 3). Paralelo a ello "son fuen- 
tes creativas, para mí son como fuentes creativas son como el hombre, son como la dualidad del hombre que se debate entre la felicidad y el dolor, básicamente es eso..." (M.F. 2). Estos señalamientos están en concordancia con los planteamientos de (Beghetto, 2014; Ivcevic y Brackett, 2015), quienes resaltan la importancia del abordaje de la relación entre creatividad y emociones.

La incidencia de las emociones en la educación es diversa, integrando aspectos tanto positivos como negativos. Particularmente sobre estos últimos Ilaman la atención los comentarios de los maestros en formación, con sus reflexiones a partir de sus propias experiencias como estudiantes, ya que como señala Zembylas (2003) la experiencia emocional de los maestros limita o potencia las posibilidades de aprendizaje de los alumnos. Es así como se sugiere que el profesorado profundice en el reconocimiento de sus propias emociones, para identificar cómo ellas inciden en sus procesos de enseñanza, pensamientos, actuaciones y en la manera de afrontar los retos, frustraciones y demás desafíos del contexto escolar (Zembylas, 2003; Zinsser et al., 2014).

A pesar de los continuos intentos por mejorar la calidad educativa e implementar reformas que generen respuesta a las actuales necesidades de los contextos educativos y sociales, los programas de formación del profesorado siguen asumiendo el desarrollo emocional de manera tímida o simplemente como un elemento que se integra, de manera implícita, en la formación pedagógica o didáctica. Este es el caso específico de los programas de licenciatura de la Facultad de Ciencias de la Educación en la UPTC, en donde es evidente que este abordaje en la mayoría de los casos no se hace de manera explícita y estructurada, ni está integrado a la configuración que se establece para la formación del profesorado. Al respecto, Bisquerra (2005) señala que el trabajo intencionado orientado a la formación de competencias emocionales en los programas de formación del profesorado, suele estar ausente o ser mínimo. En esta misma dirección, algunos participantes de este estudio, respecto al abordaje teórico plantearon: "No, como tal no se le da la importancia ni el espacio que amerita esta temática" (M.F. 6), o "Sí, en algunas clases uno lo escucha, lo ve, pero no hay nada claro" (M.F. 2); "Superficialmente, o sea, lo que yo he visto de teoría de emociones ha sido por cuenta mía, pero no que en el programa me lo den" (M.F.15).

Lo cierto es que los actuales contextos académicos, que suelen ser altamente competitivos, requieren de diversas habilidades emocionales que no suelen trabajarse, en gran medida porque se asume que el ámbito emocional y social hace parte de la intimidad de las personas (Calle et al., 2011), por lo tanto, no debe ser desarroIlado ni en la escuela, ni en la formación del profesorado. Para finalizar, el sentido y significado que los maestros en formación atribuyen a las emociones, se relaciona con el desafío que Schutz (2014) plantea respecto a la habilidad del profesorado para leer los contextos, tomar decisiones y reorientar sus acciones cuando se requiera, logrando modular la velocidad, la estrategia, la metodología y la profundidad de sus acciones, de acuerdo con las reacciones de sus estudiantes y su propio bienestar.

\section{Conclusiones}

El sentido y significado que los participantes de este estudio atribuyen a las emociones pone de manifiesto la relevancia que los maestros en formación dan a la te- 
mática y el reconocimiento de su incidencia en los procesos de enseñanza y aprendizaje. De ello resulta necesario admitir que la formación inicial del profesorado es un momento fundamental para el desarrollo de competencias emocionales, las cuales se deben seguir fomentando durante la formación continuada, logrando así mejores niveles de bienestar y éxito laboral, al igual que procesos educativos más efectivos (Palomera et al., 2008).

En los programas de formación del profesorado de la Universidad Pedagógica y Tecnológica de Colombia el abordaje de la teoría de las emociones y su relación pedagógica y didáctica es escasa y en la mayoría de las ocasiones nula, en este sentido Carpena y Darder (2010) señalan que los docentes de hoy reciben una de las mejores formaciones profesionales de toda la historia, sin embargo, no suele abordarse el ámbito intrapersonal, en particular en lo que tiene que ver con el aspecto emocional, dejando como aspectos secundarios la ilusión por la vida, el ánimo y motivación por su labor educativa, el disfrute de la interacción con los demás y los vínculos positivos con sus pares.

\section{REFERENCIAS BIBLIOGRÁFICAS}

Abarca, M., Marzo, L. y Sala, J. (2002). La educación emocional y la interacción profesor/a-alumno/a. Revista Electrónica Interuniversitaria de Formación del Profesorado, 5(3), 1-4.

Andrade, N. F. (2016). La gustatividad en la formación docente: un fenómeno afectivo emergente. Revista Electrónica de Investigación Educativa, 2(18), 92-104.

Avila, A. K. y Castañeda, D. I. (2015). Relación entre el sentido atribuido al trabajo y la conducta de compartir conocimiento. Revista Puertorriqueña de Psicología, 2(26), 238-251.

Bakker, A. B., Westman, M. y Schaufeli, W. B. (2007). Crossover of burnout: An experimental design. European Journal of Work and Organizational Psychology, 16(2), 220-239. DOI: http://doi.org/10.1080/13594320701218288.

Bar-On, R. (1997). Bar-On emotional quotient inventory: Technical manual. Toronto: Multi Health System Inc.

Bar-On, R. (2006). The Bar-On model of Emotional-Social Intelligence (ESI). Psicothema, 18(Suppl.), 13-25.

Bazarra, L., Casanova, O. y García, J. (2004). Ser profesor y dirigir profesores en tiempos de cambio. Madrid: Narcea.

Beghetto R. A. (2014). Creative mortification: An initial exploration. Psychology of Aesthetics, Creativity, and the Arts, 8(3), 266-276. DOI: http://doi.org/10.1037/ a0036618.

Bisquerra, R. (2000). Educación emocional y bienestar. Barcelona: Praxis.

Bisquerra, R. (2005). La educación emocional en la formación del profesorado. Revista interuniversitaria de formación del profesorado, 19(3), 95-114.

Bisquerra, R. y Pérez, N. (2012). Educación emocional: estrategias para su puesta en práctica. Revista de la Asociación de Inspectores de Educación de España, 16, $1-11$. 
Boyatzis, R., Goleman, D. y Rhee, K. (2000). Clustering competence in emotional intelligence: insights from the emotional competence inventory (ECI). En R. BarOn, y J. D. A. Parker (eds.), Handbook of emotional intelligence (pp. 343-362). San Francisco: Jossey-Bass.

Brackett, M. y Salovey, P. (2006). Measuring emotional intelligence with the Mayer-Salovery-Caruso Emotional Intelligence Test (MSCEIT). Psicothema, 18(Suppl.), 34-41.

Brady, S. T., Reeves, S. L., Garcia, J., Purdie-Vaughns, V., Cook, J. E., Taborsky-Barba, S., Tomasetti, S., Davis, E. M. y Cohen, G. L. (2016). The psychology of the affirmed learner: spontaneous self-affirmation in the face of stress. Journal of Educational Psychology, 3(108), 353-373. DOI: http://doi.org/10.1037/edu0000091.

Brotheridge, C. M. y Grandey, A. A. (2002). Emotional intelligence and burnout: Comparing two perspectives of "people work". Journal of Vocational Behavior, 60, 17-39. DOI: http://doi.org/10.1006/jvbe.2001.1815.

Buitrago, R. E. y Herrera, L. (2013). Matricular las emociones en la escuela, una necesidad educativa y social. Praxis \& Saber, 4(8), 87-108. DOI: http://doi. org/10.19053/22160159.2653.

Calle, M. G., Remolina, N. y Velásquez, B. M. (2011). Incidencia de la inteligencia emocional en el proceso de aprendizaje. NOVA - Publicación Científica en Ciencias Biomédicas, 15(9), 94-106.

Carpena, A. y Darder, P. (2010). Educación emocional y salud y bienestar del profesorado. En F. López (dir.), La salud física y emocional del profesorado (pp. 25-30). Barcelona: Graó.

Castro, S. A. y Zautra, A. J. (2016). Humanization of social relations: nourishing health and resilience through greater humanity. Journal of Theoretical and Philosophical Psychology, 36(2), 64-80. DOI: http://doi.org/10.1037/teo0000040.

Coleman, H. y Unrau, Y. A. (2005). En R. M. Grinnell y Y. A. Unrau (eds.), Social work: Research and evaluation. Quantitative and qualitative approaches ( $7^{\mathrm{a}} \mathrm{ed} .$, pp. 403-420). NuevaYork: Oxford University Press.

Corbin, J. y Strauss, A. (2002). Bases de la investigación cualitativa, técnicas y procedimientos para desarrollar la teoría fundamentada. Medellín: Editorial Universidad de Antioquia.

Cornejo, R. y Quiñonez, M. (2007). Factores asociados al malestar/bienestar docente. Una investigación actual. Revista electrónica iberoamericana sobre calidad, eficacia y cambio en educación, 5(5e), 75-80.

Davidson, R. J. (2003). La neurociencia de la emoción. En D. Goleman (ed.), Emociones Destructivas, cómo entenderlas y superarlas (pp. 104-118). Barcelona: Kairós.

Durán, A., Extremera, N., Rey, L., Fernández-Berrocal, P. y Montalbán F. M. (2006). Predicting academic burnout and engagement in educational settings: Assessing the incremental validity of perceived emotional intelligence beyond perceived stress and general self-efficacy. Psicothema, 18(Suppl.), 158-164.

Ekman, P. (2003). La universalidad de las emociones. En D. Goleman (ed.), Emociones Destructivas, cómo entenderlas y superarlas (pp. 71-93). Barcelona: Kairós.

Emmer, E. T. (1994). Toward an understanding of the primary of classroom management and discipline. Teaching Education, 6, 65-69. 
Eren, A. (2014). Uncovering the links between prospective teachers' personal responsibility, academic optimism, hope, and emotions about teaching: a mediation analysis. Social Psychology of Education, 17(1), 73-104. DOI: http://doi. org/10.1007/s11218-013-9243-5.

Extremera, N., Fernández-Berrocal, P. y Durán, A. (2003). Inteligencia emocional y burnout en profesores. Encuentros en Psicología Social, 1, 260-265.

Franklin, C. y Ballan, M. (2005). Reliability and validity in qualitative research. En R. M. Grinnell y Y. A. Unrau (eds.), Social work: Research and evaluation. Quantitative and qualitative approaches (7th. ed., pp. 438-449). Nueva York: Oxford University Press.

Garner, P. W., Moses, L. K. y Waajid, B. (2009). Prospective teachers' awareness and expression of emotions: associations with proposed strategies for behavioral management in the classroom. Psychology in the Schools, 5(50). DOI: http://doi. org/10.1002/pits.21688.

Goleman, D. (1998). Working with emotional intelligence. Nueva York: Bantam Books.

Goleman, D. (2001). An El-Based theory of performance. En C. Cherniss y D. Goleman (eds.), The emotionally intelligent workplace (pp. 27-44). San Francisco: Jossey-Bass.

Habók, A. y Nagy, J. (2016). In-service teachers' perceptions of project-based learning. Springer Plus, 5(83), 1-14. DOI: http://doi.org/10.1186/s40064-016-1725-4.

Hargreaves, A. (2000). Mixed emotions: Teachers' perceptions of their interactions with students. Teaching and Teacher Education, 16, 811-826. DOI: http://doi. org/10.1016/S0742-051X(00)00028-7.

Harlap, Y. (2014). Preparing university educators for hot moments: theater for educational development about difference, power, and privilege. Teaching in Higher Education, 3(19), 217-228, DOI: http://doi.org/10.1080/13562517.2013.860098.

Hernández, P. (2002). Los moldes de la mente. La Laguna: Tafor.

Hernández, R., Fernández-Collado, C. y Baptista L. (2006). Metodología de la investigación (4a ed.). México: McGraw-Hill Interamericana.

Herrera, L., Buitrago, R. E. y Avila, A. K. (2016). Empathy in future teachers of the Pedagogical and Technological University of Colombia. New Approaches in Educational Research, 1(5), 30-37. DOI: http://doi.org/10.7821/naer.2016.1.136.

Hochschild, A. R. (1990). Ideology and emotion management: A perspective and path for future research. En T. D. Kemper (ed.), Research agendas in the sociology of emotions (pp. 117-142). Albany: State University of New York Press.

Hodgen, J. y Askew, M. (2007). Emotion, identity and teacher learning: becoming a primary mathematics teacher. Oxford Review of Education 4(33), 469-487. DOI: http://doi.org/10.1080/03054980701451090.

Hué, C. (2012). Bienestar docente y pensamiento emocional. Revista Fuentes, 12, 47-68.

Hughes, B., Huston, T. y Stein, J. (2010). Using case studies to help faculty navigate difficult classroom moments. College Teaching 59(1), 7-12. DOI: http://doi. org/10.1080/87567555. 2010.489076.

Ivcevic, Z. y Brackett, M. A. (2015). Predicting creativity: interactive effects of openness to experience and emotion regulation ability. Psychology of Aesthetics, Creativity, and the Arts, 4(9), 480-487. DOI: http://doi.org/10.1037/a0039826. 
Jiboku, A. O. (2015). Effectiveness of emotional empowerment and self-efficacy techniques on occupational stress of secondary school teachers in Ogun State, Nigeria. Journal of Educational Review, 1(8), 39-46.

Klusmann, U., Richter, D. y Lüdtke, O. (2016). Teachers' emotional exhaustion is negatively related to students' achievement: evidence from a large-scale assessment study. Journal of Educational Psychology, 108(7). DOl: http://doi.org/10.1037/ edu0000125.

Macazaga, A. M., Vaquero, A. y Gómez, A. (2013). El registro de la emoción en el cuerpo, autoconocimiento y formación del profesorado. Revista Electrónica Interuniversitaria de Formación del Profesorado, 16(2), 135-145. DOI: http://doi. org/10.6018/reifop.16.2.184021.

Marchesi, Á. y Díaz, M. (2007). Sobre el bienestar de los docentes. Competencias, emociones y valores. Madrid: Alianza.

Maslach, C. (1982). Burnout: The cost of caring. Englewood Cliffs, NJ: Prentice Hall.

Mayer, J. D. y Salovey P. (1997). What is emotional intelligence? En P. Salovey y D. Sluyter (eds.), Emotional development and emotional intelligence: implications for educators (pp. 3-34). Nueva York: Basic Books.

Mesa, M. C., Lara, A. I., Herrera, L., Seijo, D., Spínola, A. y Carmona, C. (2002). La salud laboral del profesorado. Una asignatura pendiente. Granada: Grupo Editorial Universitario.

Morris, J. A. y Feldman, D. C. (1996). The dimensions, antecedents, and consequences of emotional labor. Academy of Management Review, 21, 986-1010.

Palomera, R., Fernández-Berrocal, P. y Bracket, M. (2008). La inteligencia emocional como una competencia básica en la formación inicial de los docentes: algunas evidencias. Revista electrónica de investigación psicoeducativa, 15(6) 437-454.

Palomera, R., Gil-Olarte, P. y Brackett, M. A. (2006). ¿Se perciben con inteligencia emocional los docentes? Posibles consecuencias sobre la calidad educativa. Revista de Educación, 341, 687-703.

Postareff, L. y Lindblom-Ylänne, S. (2011). Emotions and confidence within teaching in higher education. Studies in Higher Education, 7(36), 799-813. DOl: http://doi. org/10.1080/03075079.2010.483279.

Ranellucci, J., Hall, N. C. y Goetz, T. (2015). Achievement goals, emotions, learning, and performance: A process model. Motivation Science, 1(2), 98-120. DOI: http:// doi.org/10.1037/mot0000014.

Relojo, D., Pilao, S. J. y de la Rosa, R. (2015). From passion to emotion: emotional quotient as predictor of work attitude behaviour among faculty members. $I i-$ manager's Journal Psychology, 4(8), I-10.

Rodríguez, G., Gil, J. y García, E. (1996). Metodología de la investigación cualitativa. Archidona (Málaga): Ediciones Aljibe.

Schaubroeck, J. y Jones, J. R. (2000). Antecedents of workplace emotional labor dimensions and moderators of their effects on physical symptoms. Journal of Organizational Behavior 21(2), 163-183. DOI: http://doi.org/10.1002/(SICI)10991379(200003)21:2<163::AID-JOB37>3.0.CO;2-L. 
Schutz, P. A. (2014). Inquiry on teachers' emotion. Educational Psychologist, 49(1), 1-12. DOI: http://doi.org/10.1080/00461520.2013.864955.

Servan-Scheiber, D. (2004). Curación emocional. Barcelona: Kairós.

Sue, D. W., Lin, A. I., Torino, G. C., Capodilupo, C. M. y Rivera, D. P. (2009). Racial microaggressions and difficult dialogues on race in the classroom. Cultural Diversity and Ethnic Minority Psychology 15(2), 183-190. DOI: http://doi.org/10.1037/ a0014191.

Warren, L. (2006). Managing hot moments in the classroom. Cambridge, MA: Derek Bok Center for Teaching and Learning.

Yin, R. K. (1994). Case study research: design and methods, applied social research (2nd. ed.). Newbury Park, CA: Sage.

Yin, R. K. (1999). Enhancing the quality of case studies in health services research. Health Services Research, 34, 1209-1224.

Zee, M., de Jong, P. F. y Koomen, H. M. Y. (2016). Teachers' self-efficacy in relation to individual students with a variety of social-emotional behaviors: a multilevel investigation. Journal of Educational Psychology, 108(7), 1013-1027. DOI: http:// doi.org/10.1037/edu0000106.

Zembylas, M. (2003). Caring for teacher emotion: Reflections on teacher self development. Studies in Philosophy and Education, 22, 103-125. DOI: http://doi. org/10.1023/A:1022293304065.

Zinsser, K. M., Shewark, E. A., Denham, S. A. y Curby, T. W. (2014). A mixed-method examination of preschool teacher beliefs about social-emotional learning and relations to observed emotional support. Infant and Child Development, 23, 471493. DOI: http://doi.org/10.1002/icd.1843. 
